\title{
Técnica de centrífugo-flutuação com sulfato de zinco no diagnóstico de helmintos gastrintestinais de gatos domésticos
}

\author{
Zinc sulfate centrifugal flotation technique on the diagnosis of gastrointestinal helminthes \\ of domestic cats
}

\begin{abstract}
Letícia Mattos de Souza-Dantas ${ }^{{ }^{*}}$ Otílio Pereira Machado Bastos ${ }^{\text {II }}$ Beatriz Brener $^{\text {II }}$ Márcia Salomão ${ }^{\text {III }}$ Jorge Guerrero $^{\text {IV }}$ Norma Vollmer Labarthe ${ }^{\mathrm{I}}$
\end{abstract}

\section{- NOTA -}

\section{RESUMO}

O diagnóstico coproparasitológico é o recurso laboratorial mais utilizado para detecção de infecções parasitárias gastrintestinais. A técnica de centrífugo-flutuação com sulfato de zinco (FAUST et al., 1938) é técnica de eleição no diagnóstico de estruturas parasitárias leves, podendo também ser usada para detecção de estruturas pesadas. Para avaliar o desempenho dessa técnica no diagnóstico das helmintoses gastrintestinais de gatos domésticos, foram realizados exames coproparasitológicos de 13 gatos domésticos 15 dias antes de suas mortes. À necropsia, os helmintos adultos encontrados no tubo digestivo e fígado foram contados, fixados e identificados. A técnica utilizada mostrou-se capaz de recuperar ovos de todos os nematóides e trematódeos encontrados, mas não foi adequada para a recuperação de cestóides.

Palavras-chave: parasitologia, exame coproparasitológico, parasitos gastrintestinais.

\section{ABSTRACT}

Fecal examinations are the most commonly used laboratory tool for diagnosis of gastrointestinal nematode parasitic infections. Zinc centrifugal floatation technique (FAUST et al., 1938) is not only considered to be efficient in detecting light parasitic structures, but it is also used for recovery of heavy structures. In order to evaluate the performance of that technique in the diagnosis of domestic cats' gastrointestinal helminthiasis, fecal examinations of 13 animals were carried out 15 days prior to the animals' deaths. At necropsy, adult gastrointestinal parasites found were collected, counted, fixed and identified. The studied technique was able to recover eggs from all detected nematodes and trematodes, but appeared inappropriate for recovery of cestode eggs.

Key words: parasitology, fecal examination, gastrointestinal parasites.

As helmintíases gastrintestinais são uma importante causa de morbidade de animais domésticos e o diagnóstico coproparasitológico ainda é o recurso laboratorial mais utilizado, por ser de fácil execução e baixo custo (MELLO et al., 1989; WILCOX \& COURA, 1991).

A técnica de centrífugo-flutuação com sulfato de zinco (Técnica de Faust) (FAUST et al., 1938) é técnica de escolha na detecção de estruturas leves (DUBEY, 1993), podendo também ser usada na detecção de ovos pesados (CARLETON \& TOLBERT, 2004), o que sugere ser uma técnica com boa sensibilidade diagnóstica (DRYDEN, 2005; FAUST et al., 1938). Assim, avaliou-se a eficácia do uso da técnica de Faust no diagnóstico coproparasitológico de helmintíases em gatos domésticos.

Foram estudados 13 gatos domésticos, independentemente de patologias concomitantes, da região metropolitana do Rio de Janeiro, no período de

IPrograma de Pós-graduação em Medicina Veterinária, Faculdade de Veterinária, Universidade Federal Fluminense (UFF), Niterói,

RJ, Brasil. Rua Maria da Ajuda Medeiros, n. 31, casa 1, Itaipu, 24340-170, Niterói, RJ, Brasil. E-mail: souza_dantas@yahoo.com.br.

* Autor para correspondência.

IIDepartamento de Microbiologia e Parasitologia, Instituto Biomédico, UFF, Niterói, RJ, Brasil.

IIIDepartamento de Patologia e Clínica Veterinária, Faculdade de Veterinária, UFF, Niterói, RJ, Brasil.

${ }^{\mathrm{IV}}$ Department of Pathobiology, School of Veterinary Medicine, University of Pennsylvania, Philadelphia, PA, USA. 
março a abril de 2004. Os animais incluídos no estudo tinham mais de seis meses de idade, sem distinção de sexo ou raça, e tiveram, pelo menos, uma amostra de fezes colhida por seus responsáveis até 15 dias antes da morte, natural ou por eutanásia. As eutanásias foram realizadas pelos médicos veterinários responsáveis pelos animais, utilizando acepromazina $\left(0,2 \mathrm{mg} \mathrm{kg}^{-1} \mathrm{IM}\right)$, tiopental (25mg $\left.\mathrm{kg}^{-1} \mathrm{IV}\right)$ e cloreto de potássio a $10 \%$ (2mmol kg-1 IV) (BEAVER et al., 1981; CFMV, 2002). Os cadáveres eram recebidos sob refrigeração e as necropsias realizadas em até 24 horas após a morte.

As amostras de fezes foram mantidas a $4^{\circ} \mathrm{C}$, até o processamento, em no máximo 12 horas, pela técnica de Faust, utilizando-se solução aquosa de sulfato de zinco com densidade de $1.200 \mathrm{~g} \mathrm{~cm}^{-3}$ (FAUST et al., 1938). Os responsáveis pelos animais coletaram as amostras em até oito horas após a defecação. Foi preparada uma lâmina para cada amostra fecal, com material obtido da película superficial originada após centrifugação, utilizando-se alça de platina, e montada com lamínula medindo 20X20mm. As lâminas foram sistematicamente examinadas à microscopia óptica, todas as estruturas parasitárias encontradas foram identificadas e contadas, e os resultados anotados em fichas individuais dos animais. Quando mais de uma amostra do mesmo animal foi submetida, trabalhou-se com a média do número de ovos encontrados.

O procedimento necroscópico parasitológico foi realizado segundo SALOMÃO (2005). Os parasitos encontrados foram imediatamente fixados em solução de AFA (álcool 70-fomol-ácido acético) a quente, seguindo-se etapas de fixação padrão para nematóides, cestóides e trematódeos (GOULART \& LEITE, 1978). Os indivíduos encontrados foram identificados por espécie, pelas chaves de VICENTE et al. (1997), TRAVASSOS et al. (1969) e YAMAGUTI (1962).

Como não foi possível fazer a especificação de ancilostomídeos pela observação dos ovos (BOWMAN, 2003), optou-se por comparar os resultados de coproparasitologia com o gênero dos parasitos adultos. Além disso, há controvérsia quanto à taxonomia das espécies de trematódeos do gênero Platynosomum, o que fez com que também fossem identificados apenas pelo gênero (SALOMÃO, 2005).

A sensibilidade e a especificidade dos exames realizados pela técnica de Faust foram calculadas utilizando-se os resultados da pesquisa de helmintos à necropsia como padrão ouro (PEREIRA, 1995)

Dentre os 13 animais examinados, apenas um não teve nenhuma helmintíase detectada, nem à necrópsia e nem ao exame coproparasitológico. Nos demais, foram encontradas quatro espécies de nematóides (Toxocara cati, Toxascaris leonina,
Ancylostoma tubaeforme e Ancylostoma braziliense), uma de cestóide (Dipylidium caninum) e uma de trematódeo (Platynosomum sp.) (Tabela 1).

Na recuperação de ovos de Ancylostoma, houve concordância entre os exames coproparasitológicos e necroscópicos quando o número de ovos identificados por lâmina observada foi elevado $(\geq 150)$. Entretanto, quando o número de ovos contados foi baixo ( $<15)$, não se encontrou formas adultas à necropsia, tanto assim que a sensibilidade foi de $100 \%$ e a especificidade de $44 \%$. O número de falso positivos pode ter sido elevado em conseqüência da grande capacidade de oviposição das fêmeas do gênero Ancylostoma (BOWMAN, 2003), propiciando o achado de ovos mesmo em presença de cargas parasitárias muito baixas. Por outro lado, a carga parasitária baixa, associada ao tamanho diminuto das formas adultas, podem ter contribuído para a perda de alguns helmintos durante os procedimentos de necropsia. Além disso, como as amostras eram coletadas pelos responsáveis pelos animais, pode ter havido contaminação pelo contato com fezes de outros animais, ou ainda veiculado por fômites (Tabela 1).

Houve concordância entre os achados de ovos nas fezes e de parasitos no tubo digestivo, no caso de T.leonina (sensibilidade e especificidade = 100\%) (Tabela 1). Quanto à T.cati, houve duas exceções: um gato albergava um único exemplar macho e, em suas fezes, não se encontrou ovos; e, outro gato, no qual foi encontrado apenas um ovo e não foi identificado nenhum adulto à necropsia (sensibilidade $=100 \% \mathrm{e}$ especificidade $=91 \%$ ) $($ Tabela 1$)$.

Embora houvesse gatos infectados por D.caninum, não foi possível detectar ovos ou cápsulas ovígeras nas fezes (Tabela 1), indicando que a técnica de Faust não é adequada para diagnóstico de infecções por esse cestóide (VISCO et al., 1978). O parasito que mostrou menor regularidade entre sua ocorrência e o achado de ovos nas fezes foi Platynosomum sp. (sensibilidade $=60 \%$ e especificidade $=88 \%$ ) $($ Tabela 1). Cabe lembrar que a pesquisa de parasitos de localização hepática é difícil, o que pode ter favorecido a perda dos indivíduos quando a carga parasitária era baixa, e que a produção de ovos por esses parasitos é baixa e intermitente (PALUMBO et al., 1976), gerando baixa densidade de ovos nas fezes dos animais parasitados.

A técnica de Faust foi capaz de recuperar ovos de todos os nematóides e trematódeos encontrados, com maior ou menor acurácia, na dependência da espécie em questão, podendo ser útil ao diagnóstico das infecções por nematóides e trematódeos de gatos domésticos. Entretanto, não se mostrou apropriada para o diagnóstico de infecções pelo cestóide D.caninum. 
Tabela 1 - Achados de ovos de helmintos em exames coproparasitológicos realizados pela técnica de centrífugo-flutuação com sulfato de zinco e número de parasitos encontrados à necropsia de gatos domésticos da Região Metropolitana do Rio de Janeiro, R.J.

\begin{tabular}{|c|c|c|c|c|c|c|c|c|c|c|}
\hline \multirow{2}{*}{ Gatos } & \multicolumn{2}{|c|}{ Ancylostoma spp. } & \multicolumn{2}{|c|}{ Toxocara cati } & \multicolumn{2}{|c|}{ Toxascaris leonina } & \multicolumn{2}{|c|}{ Dipylidium caninum } & \multicolumn{2}{|c|}{ Platynosonum sp. } \\
\hline & Ovos & Adultos & Ovos & Adultos & Ovos & Adultos & Ovos & Adultos & Ovos & Adultos \\
\hline 1 & + & 0 & + & 10 & - & 0 & - & 0 & + & 47 \\
\hline 2 & - & 0 & - & 0 & - & 0 & - & 0 & - & 0 \\
\hline 3 & + & 80 & - & 0 & - & 0 & - & 0 & + & 142 \\
\hline 4 & + & 17 & - & 0 & - & 0 & - & 6 & - & 0 \\
\hline 5 & + & 49 & + & 0 & - & 0 & - & 0 & + & 9 \\
\hline 6 & + & 212 & - & 0 & - & 0 & - & 0 & - & 0 \\
\hline 7 & + & 0 & + & 9 & + & 3 & - & 0 & + & 0 \\
\hline 8 & - & 0 & - & 0 & - & 0 & - & 42 & - & 0 \\
\hline 9 & + & 0 & - & 0 & + & 3 & - & 0 & - & 0 \\
\hline 10 & + & 0 & - & 1 & + & 26 & - & 0 & - & 10 \\
\hline 11 & + & 0 & - & 0 & - & 0 & - & 0 & - & 0 \\
\hline 12 & - & 0 & - & 0 & - & 0 & - & 2 & - & 5 \\
\hline 13 & - & 0 & - & 0 & - & 0 & - & 11 & - & 0 \\
\hline
\end{tabular}

(-) Ausência de ovos no exame microscópico.

(+) Presença de ovos no exame microscópico.

\section{REFERÊNCIAS}

BEAVER, B.B. et al. Report of the AVMA panel on euthanasia. Journal of American Veterinary Medical Association, v.218, n.5, p.671-696, 1981.

BOWMAN, D.D. Diagnostic parasitology. In: Geogis' parasitolgy for veterinarians. 8.ed. St.Louis: Saunders, 2003. Cap.5, p.287-358.

CARLETON, R.E.; TOLBERT, M.K. Prevalence of Dirofilaria immitis and gastrointestinal helminthes in cats euthanized at animal control agencies in northwest Georgia. Veterinary Parasitology, v.119, n.4, p.319-326, 2004.

CONSELHO FEDERAL DE MEDICINA VETERINÁRIA (CFMV). Procedimentos e métodos de eutanásia em animais. Resolução no ${ }^{\circ} .714$ de 20 de junho de 2002. Acesso em: 07 mar 2005. On line. Disponível em: <http:// www.cfmv.org.br>.

DRYDEN, M.W. Comparison of common fecal flotation techniques for the recovery of parasite eggs and oocysts. Veterinary Therapeutics, v.6, n.1, p.15-28, 2005.

DUBEY, J.P. Intestinal protozoa infections. Veterinary Clinics of North América: Small Animal Practice, v.23, n.1, p.37-55, 1993.

FAUST, E.C. et al. A critical study of clinical laboratory technics for the diagnosis of protozoan cysts and helminth eggs in feces I. Preliminary communication. American Journal of Tropical Medicine, v.18, p.169-183, 1938.

GOULART, E.G.; LEITE, I.C. Técnicas helmintológicas. In:_____. Moraes parasitologia e micologia humana. 2.ed. Rio de Janeiro: Cultura Médica, 1978. p.523-528.

MELLO, R.T. et al. Estudo comparativo entre os métodos "Coprotest" e de Hoffman, Pons e Janer no diagnóstico de parasitoses intestinais. Revista de Farmácia e Bioquímica da Universidade Federal de Minas Gerais, v.10, p.9-15, 1989.

PALUMBO, N.E. et al. Evaluation of fecal technics for the diagnosis of cat liver fluke infection. Laboratory Animal Science, v.26, n.3, p.490-493, 1976.

PEREIRA, M.G. Epidemiologia - teoria e prática. Rio de Janeiro: Guanabara Koogan, 1995. 596p.

SALOMÃO, M.C.S. et al. Ultrasonography in hepatobiliary evaluation of domestic cats (Felis catus L., 1758) infected by Platynosomum Looss, 1907. International Journal of Applied Research in Veterinary Medicine, v.3, n.3, p.271279, 2005.

TRAVASSOS, L. et al. Trematódeos do Brasil. Memórias do Instituto Oswaldo Cruz, v.67, p.140-141, 1969. (fascículo único).

VICENTE, J.J. et al. Nematóides do Brasil, Parte V: nematóides de mamíferos. Revista Brasileira de Zoologia, v.14, suplem.1, p.1-452, 1997.

VISCO, R.J. et al. Effect of age and sex on the prevalence of intestinal parasitism in cats. Journal of American Veterinary Medical Association, v.172, n.7, p.797-800, 1978.

WILLCOX, H.P.; COURA, J.R. The efficiency of Lutz, KatoKatz and Baermann-Moraes (adapted) techniques association to the diagnosis of intestinal helmints. Memórias do Instituto Oswaldo Cruz, v.86, n.4, p.457-460, 1991.

YAMAGUTI, S. Systema Helminthum. In: Cestodes of vertebrates. New York: Interscience, 1962. V.2, parte 5, Cap.3, p.350-449. 